\title{
Densidade ótima e aceitável de estradas na colheita de pinus no sistema de toras curtas e árvores inteiras
}

\author{
Optimum and acceptable forest road density in pine \\ harvesting for cut-to-length and full tree systems
}

\author{
Franciny Lieny Souza ${ }^{1}$, Jean Alberto Sampietro르, Helen Michels Dacoregio', \\ Philipe Ricardo Casemiro Soares ${ }^{2}$, Eduardo da Silva Lopes ${ }^{3}$ e Dagoberto Stein Quadros ${ }^{4}$
}

\section{Resumo}

O objetivo deste trabalho foi determinar o ponto economicamente ótimo e aceitável de densidade de estradas para povoamentos de Pinus taeda L. submetidos ao corte raso em diferentes sistemas de colheita da madeira. O estudo foi realizado em uma empresa na região do Planalto Serrano de Santa Catarina, em povoamentos aos 33 anos de idade submetidos ao corte raso no sistema de colheita de toras curtas (CTL) e árvores inteiras (FT). No sistema CTL, as operações de extração foram realizadas utilizando forwarder, enquanto no FT foi utilizado o skidder. A densidade ótima de estradas (DOE) foi calculada pelo método indireto, sendo determinados os custos de extração da madeira para ambos os sistemas, os custos de reconstrução e manutenção de estradas e de perda de área produtiva. A densidade aceitável (DAE) foi determinada como sendo uma variação de $2 \%$ acima do custo total mínimo, conforme Malinovski (2013). Para ambos os sistemas de colheita da madeira, a densidade atual de estradas $(92,02 \mathrm{~m} / \mathrm{ha})$ foi superior à DOE e DAE, que foi de 15,63 e $24,75 \mathrm{~m} / \mathrm{ha}$, no CTL e de 22,85 e $27,00 \mathrm{~m} / \mathrm{ha}$ no FT, respectivamente. Tanto na DOE e DAE, os custos com extração foram maiores no sistema CTL, embora os custos com reconstrução e manutenção de estradas e perda de área produtiva foram menores que o sistema FT. A consideração da DOE no planejamento das operações florestais possibilitará a redução do custo total em $33 \%$ no sistema CTL e de $54 \%$ no sistema FT, possibilitando a otimização dos recursos e maximização das receitas.

Palavras-chave: Colheita florestal; planejamento de estradas; otimização, custos.

\begin{abstract}
The objective of this work was to determine the optimal and acceptable economically point of road density for Pinus taeda L. stands in clear cut operations in different harvesting systems. The study was conducted in a forest company in the Mountain Plateau regions of Santa Catarina State, in areas with 33 years old stands which were submitted to clear cut in cut-to-length (CTL) and full tree (FT) harvesting systems. In the CTL systems, the extraction operation was performed using forwarder, whereas in FT system skidder was used. The optimal road density (DOE) was calculated by the indirect method, where the costs of wood extraction for both systems were determined, as well as for roads reconstruction, roads maintenance and productive area loss. The acceptable road density (DAE) was determined, being $2 \%$ above the minimum total cost, according to Malinovski (2013). For both systems, the current road density $(92.02 \mathrm{~m} / \mathrm{ha})$ was higher than the DOE and DAE, which was 15.63 and $24.75 \mathrm{~m} /$ ha, respectively, for the CTL system and 22.85 and $27.00 \mathrm{~m} / \mathrm{ha}$, respectively, for the FT system. Both in the DOE and DAE the extraction costs were higher in the CTL system, although the costs with roads reconstruction and maintenance and productive area loss were lower than the FT system. Thus, with the use of the DOE, it will be possible to reduce the total cost by $33 \%$ in the CTL system and by $54 \%$ in the FT system. Therefore, it is recommended to use DOE or DAE to re-plan operations, aiming at resources optimizing and maximizing revenues.
\end{abstract}

Keywords: forest harvesting; forest roads; extraction operations; optimal forest roads.

\footnotetext{
${ }^{1}$ Mestre em Engenharia Florestal. UDESC - Universidade do Estado de Santa Catarina / Centro Agroveterinário. Avenida Luiz de Camões - Conta Dinheiro - 88520000 - Lages, SC, Brasil. E-mail: francinylieny@hotmail.com, helenmdacoregio@gmail.com.

2Professor Adjunto do Departamento de Engenharia Florestal. UDESC - Universidade do Estado de Santa Catarina / Centro Agroveterinário. Avenida Luiz de Camões - Conta Dinheiro - 88520000 - Lages, SC, Brasil. E-mail: engsampietro@gmail.com,prcsoares@gmail.com.

3Professor Associado do Departamento de Engenharia Florestal. UNICENTRO - Universidade Estadual do Centro-Oeste / Centro de Ciências Agrárias e Ambientais. PR 153, KM 7 - Cx. Postal 21 - 84500000 - Irati, PR, Brasil - Caixa-postal: 21. E-mail: eslopes@irati.unicentro.br

${ }^{4}$ Professor Titular do Departamento de Engenharia Florestal. FURB - Fundação Universidade Regional de Blumenau / Centro de Ciências Tecnológicas. Rua São Paulo - 3250 - 89030000 - Blumenau, SC, Brasil. E-mail: engdagobert@gmail.com.
}

Sci. For., Piracicaba, v. 46, n. 118, p. 189-198, jun. 2018 DOI: dx.doi.org/10.18671/scifor.v46n118.05 


\section{INTRODUÇÃO}

A rede viária é um elemento fundamental na implantação de um empreendimento florestal, e por isso, o seu planejamento deve ser realizado buscando fornecer o acesso às áreas de produção, permitindo o escoamento da madeira de forma eficiente, a otimização dos processos, minimização de custos e atendimento às demandas operacionais, além de contemplar questões sociais e ambientais das áreas (SOUZA, 2016). Durante a colheita e transporte da madeira, a rede viária assume destaque, pois influencia significativamente no valor final do produto (ZAGONEL et al., 2008), uma vez que uma maior extensão e qualidade de estradas permite uma redução nos custos de produção, porém, a construção de elevada densidade de estrada, pode ocasionar um investimento excessivo e perda de área produtiva.

Em geral, o planejamento da rede viária florestal se dá antes da implantação do povoamento, momento onde define-se a densidade em cada área, a qual é expressa pela relação existente entre a extensão das estradas e a área de plantio. Dessa forma, se torna fundamental caracterizar a intensidade da construção de estradas, indicando a viabilidade da rede que está sendo planejada (MACHADO, 2013). Para qualquer local há uma densidade ótima de estradas (DOE) que minimiza o custo de construção, manutenção e extração de madeira (RYAN et al. 2004), podendo esta ser definida como uma técnica quantitativa que estabelece a extensão de estradas para uma determinada área, onde a vantagem de uma rede menos densa é avaliada comparando o aumento nos custos da colheita de madeira com as economias obtidas na construção e manutenção das estradas (MACHADO, 2013).

A DOE pode variar de acordo com o manejo da floresta, com as condições do terreno e com o sistema de colheita de madeira adotado (LOPES et al., 2002). A densidade de estradas tende a ser maior a medida em que a declividade do terreno aumenta, já que em maiores declividades, as máquinas de colheita tendem a ser menos eficientes. Em regime de desbastes, os custos com manutenção tendem a ser mais elevados, devido ao maior número de intervenções na floresta e à maior dificuldade de secagem das estradas em condições climáticas adversas. Em regime de corte raso, mesmo que o volume transportado seja maior, os custos com manutenção tendem a ser menores, devido a facilidade de secagem das estradas com auxílio de faixa de insolação.

O sistema de colheita influencia a DOE principalmente devido à distância de extração interferir diretamente na produtividade das máquinas. Em sistema de toras curtas, espera-se que a densidade ótima de estradas seja inferior quando comparado ao sistema de árvores inteiras, pois, em sistemas de toras curtas, geralmente são empregadas máquinas que realizam a extração da madeira por baldeio, como o forwarder, o qual tem maior parte do tempo do ciclo operacional despendido com atividades de carregamento e descarregamento da madeira (LOPES et al., 2016; SEIXAS, 2008; MINETTE et al., 2004), havendo, dessa forma, menor influência da distância de extração na produtividade e, consequentemente, a densidade de estrada ótima tende a ser menor. Já em sistemas de colheita de árvores inteiras, geralmente são empregadas máquinas que realizam a extração por arraste, como o skidder, o qual tende a despender maior parte do ciclo operacional com atividades de deslocamento (BIRRO et al., 2002; SEIXAS, 2008) e, dessa forma, sua produtividade é mais fortemente influenciada pela distância de extração, tornando a operação mais dependente de uma maior densidade de estradas.

Entretanto, o uso da densidade economicamente ótima de estradas como parâmetro para o planejamento deve ter ressalvas, pois, em muitas empresas o emprego da DOE se torna inviável devido à rede de estradas já estar definida. Nesses casos, o planejamento da reconstrução e manutenção das estradas existentes pode ser baseado em uma densidade que pode ser considerada aceitável. Malinovski (2013) em seu trabalho considerou que a densidade aceitável de estradas (DAE) é um valor percentual acima do custo total mínimo resultante da soma dos custos com construção e manutenção, extração e perda de área produtiva.

Dessa forma, devido à densidade de estradas ter grande influência operacional e interferir diretamente no desempenho financeiro geral das empresas florestais, o uso de parâmetros como a DOE e DAE subsidia a tomada de decisão, visando à melhoria da aplicação de recursos, redução de custos em busca da sustentabilidade florestal. Nesse contexto, objetivou-se com este trabalho, determinar o ponto economicamente ótimo e aceitável na densidade de estradas em povoamentos florestais de Pinus taeda L. em corte raso em sistema de toras curtas e árvores inteiras. 


\section{MATERIAL E MÉTODOS}

\section{Caracterização da área de estudo}

O trabalho foi realizado em áreas operacionais com povoamentos de Pinus taeda L. de uma empresa florestal localizada no Planalto Serrano de Santa Catarina. Os povoamentos na ocasião do corte raso tinham 33 anos de idade, densidade média de 268,00 árv/ha, diâmetro à altura do peito médio de 43,30 cm, altura total média de 32,00 m e volume médio individual de 2,12 $\mathrm{m}^{3} \mathrm{cc} / a$ av. O relevo da área de estudo era forte ondulado, com declividade média de $15,23^{\circ}$, com talhões de aproximadamente 4,23 ha e distância de extração média de 64,56 m.

Nas operações de colheita da madeira foram avaliados dois sistemas de colheita da madeira, sendo o primeiro de toras curtas (CTL) composto por harvester que realizava as etapas de corte e por forwarder (especificações técnicas na tabela 1) que realizava a extração da madeira por baldeio; e o segundo, um sistema de árvores inteiras (FT) composto por feller direcional que realizava a derrubada das árvores, por skidder (especificações técnicas na tabela 1) que realizava a extração das árvores por arraste, e por harvester que realizava o processamento das árvores na margem da estrada. Em ambos os sistemas eram produzidos diversos sortimentos de tora com volume variando de 0,069 a $0,390 \mathrm{~m}^{3} \mathrm{cc}$.

Tabela 1. Especificações técnicas das máquinas de extração.

Table 1. Technical specifications of the extraction machines.

\begin{tabular}{lcccccc}
\hline \multirow{2}{*}{$\begin{array}{l}\text { Situação } \\
\text { operacional }\end{array}$} & Máquina & Potência & $\begin{array}{c}\text { Capacidade do tanque } \\
\text { de combustível (L) }\end{array}$ & $\begin{array}{c}\text { Alcance máximo } \\
\text { do implemento }\end{array}$ & $\begin{array}{c}\mathbf{N}^{\circ} \text { de } \\
\text { pneus }\end{array}$ & $\begin{array}{c}\text { Peso Bruto } \\
\text { total (Kg) }\end{array}$ \\
\hline \cline { 2 - 7 } Corte raso - CTL & 1910 & $249 \mathrm{cv}(186 \mathrm{~kW})$ & 184 & $7,20 \mathrm{~m}$ & 8 & 21.800 \\
Corte raso - FT & $748 \mathrm{H}$ & $193 \mathrm{hp}(144 \mathrm{~kW})$ & 297 & $3,23 \mathrm{~m}$ & 4 & 17.028 \\
\hline
\end{tabular}

Em relação às estradas, foram avaliadas as operações de reconstrução e manutenção de estradas florestais, pois não haviam operações de construção de estradas, uma vez que a rede viária na empresa já estava consolidada. As operações de reconstrução eram realizadas em torno de três meses antes do início das operações de colheita e transporte, sendo composta pelas atividades de regularização do leito da estrada, exploração e transporte de material rochoso, revestimento e fechamento da área (pós colheita). Já as operações de manutenção eram executadas durante as operações de colheita e transporte, sendo composta pelas atividades de regularização do leito da estrada, exploração e transporte de material rochoso e revestimento do leito.

Devido à especificidade do uso e reconstrução das estradas primárias, as avaliações realizadas no presente estudo contemplaram somente as estradas florestais secundárias e terciárias. As estradas secundárias possuíam largura média de 5,5 m, eram revestidas e com uso intermitente, enquanto as estradas terciárias possuíam largura média de 3,5 m, eram revestidas em pontos específicos e com uso sazonal.

A densidade atual de estradas (DE), considerada antes do estudo e obtida pela razão da quantidade total de estradas, secundárias e terciárias, em metros pela área de plantio, era de 92,02 metros lineares por hectare (m/ha), sendo a proporção da área de efetivo plantio ocupada por estradas de $4,14 \%$.

\section{Determinação da densidade ótima de estradas florestais (DOE) e densidade aceitável de estradas (DAE)}

A determinação da DOE foi realizada utilizando o método indireto, baseado no trabalho de Zagonel et al. (2008) e a DAE conforme a metodologia proposta por Malinovski (2013). Os custos com reconstrução foram calculados a partir do somatório dos custos de depreciação e de oportunidade, sendo considerado um intervalo de 8 anos (período médio entre o corte raso e o primeiro desbaste) para cálculo da depreciação, conforme a equação a seguir:

$$
\mathrm{DP}=\frac{\mathrm{COR}}{\mathrm{IT}}
$$

Em que: $D P=$ Depreciação $(R \$ / m) ; C O R=$ Custo com operações de reconstrução $(R \$ / m) ; I T=I n t e r-$ valo entre operações (anos). 
Considerou-se como custo de oportunidade os custos referentes ao uso da terra e de capital empregado na reconstrução da estrada. O custo de oportunidade do capital foi calculado de acordo com a fórmula da USDA (2001):

$$
\operatorname{Coc}=\frac{V i}{2} * i
$$

Em que: $C o c=$ custo anual de oportunidade do capital $(R \$ / m . a n o) ; V i=$ valor de investimento inicial (ou de reconstrução) $(R \$ / m) ; V f=$ valor final do investimento $(R \$ / m) ; i=$ taxa de juros ( $4 \%$ a.a.).

Com a determinação dos custos de depreciação e de oportunidade do capital em $\mathrm{R} \$$ por metro linear, os mesmos foram convertidos em $\mathrm{R} \$$ por metro cúbico de madeira com casca $\left(\mathrm{R} \$ / \mathrm{m}^{3} \mathrm{cc}\right)$, pela seguinte fórmula:

$$
\mathrm{CC}=\frac{(\mathrm{DP}+\mathrm{Coc}) * \mathrm{DE} * \mathrm{~A}}{\mathrm{~V}}
$$

Em que: $C C=$ Custo com reconstrução $\left(R \$ / m^{3} c c\right) ; D P=$ Custo de depreciação $(R \$ / m) ; C o c=$ Custo de oportunidade do capital $(R \$ / \mathrm{m}) ; D E=$ Densidade de estradas $\left(\mathrm{m} / \mathrm{m}^{2}\right) ; A=$ área total com povoamento $e$ estradas $\left(\mathrm{m}^{2}\right) ; V=$ Volume total da área $\left(\mathrm{m}^{3} \mathrm{cc}\right)$.

Os custos relacionados às atividades de manutenção não foram depreciados, pois se considerou que os mesmos são para aquele período específico, sendo calculados de acordo com a fórmula:

$$
\mathrm{CM}=\frac{\mathrm{COM} * \mathrm{DE} * \mathrm{~A}}{\mathrm{~V}}
$$

Em que: $C M=$ Custo de manutenção de estradas $\left(R \$ / m^{3} c c\right) ; C O M=$ Custo com operações de manutenção $(R \$ / \mathrm{m}) ; D E=$ Densidade de estradas $\left(\mathrm{m} / \mathrm{m}^{2}\right) ; A=$ área total com povoamento e estradas $\left(\mathrm{m}^{2}\right) ; V=$ Volume total da área $\left(\mathrm{m}^{3} \mathrm{cc}\right)$.

Os custos relacionados à perda de área produtiva corresponderam ao valor da madeira que seria produzido anualmente nas áreas ocupadas pelas estradas. Para os cálculos utilizou-se as informações obtidas com a empresa sobre o preço da terra útil para plantio $(4.861,00 \mathrm{R} \$ / \mathrm{ha})$, volume de madeira por hectare $\left(614,00 \mathrm{~m}^{3} \mathrm{cc} / \mathrm{ha}\right)$ e preço médio da madeira em pé $\left(177,70 \mathrm{R} \$ / \mathrm{m}^{3} \mathrm{cc}\right)$. Assim, o valor da terra na área com estradas foi obtido pelo produto do preço da terra e a área ocupada pelas estradas:

$$
\text { Cte }=\mathrm{Pt} * \mathrm{Ae}
$$

Em que: Cte: Custo da terra na área com estradas (R\$); Ae: Área ocupada pelas estradas (ha); Pt: Preço da terra $(R \$ / h a)$.

Aplicou-se a seguinte fórmula para o cálculo da receita total nas áreas ocupadas com estradas:

$$
\text { Rte }=\mathrm{Ae} * \mathrm{~V} * \text { Pme }
$$

Em que: Rte: Receita total na área com estradas (R\$); Ae: Área ocupada pelas estradas (ha); V: Volume de madeira $\left(\mathrm{m}^{3} \mathrm{cc} / \mathrm{ha}\right)$; Pme: Preço da madeira em pé $\left(R \$ / \mathrm{m}^{3} \mathrm{cc}\right)$.

Para a obtenção do custo anual de perda de área de produtiva, foi realizado um fluxo de caixa, considerando como custo de implantação do povoamento no ano 1 o custo da terra, e como receitas as obtidas nas áreas ocupadas com estradas nos anos de intervenção 8, 13, 18 e 25. Depois de realizado o fluxo de caixa, obteve-se o valor presente líquido (VPL) total da perda de área produtiva, utilizando uma taxa de juros de $4 \%$ (utilizado pela empresa). Para a transformação na unidade requerida $\left(\mathrm{R} \$ / \mathrm{m}^{3} \mathrm{cc}\right)$, dividiu-se o valor pelo volume total a ser colhido em cada área.

Para os custos referentes às operações de extração, primeiramente, foram obtidos dados de produtividade dos equipamentos por meio de estudo de tempos e movimentos pelo método de cronometragem de tempo individual. A produtividade encontrada foi para a situação específica do local onde a máquina estava trabalhando, e por isso, para a estimativa da produtividade em função da variação das variáveis distância, declividade volume por tora e volume por ciclo, para o forwarder e distância e declividade para o skidder, prosseguiu-se com o ajuste de equações de regressão para estimativa da produtividade em função da distância de extração. Assim, os custos de extração foram calculados pela razão entre o custo operacional total ( $\mathrm{R} \$ / \mathrm{he}$ ) e a produtividade $\left(\mathrm{em} \mathrm{m}^{3} \mathrm{cc} / \mathrm{he}\right)$ dos equipamentos de cada sistema de colheita da madeira, obtendo-se o custo de produção $\left(\mathrm{R} \$ / \mathrm{m}^{3} \mathrm{cc}\right)$, que foi relacionado com distância de extração e, consequentemente, com a densidade de estradas.

Para determinação da DOE, primeiramente, foi feito o somatório dos custos com reconstrução e 
manutenção de estradas, perda de área produtiva e extração, sendo estes relacionados com a densidade de estradas, conforme a fórmula a seguir:

$$
\mathrm{CT}=\mathrm{CC}+\mathrm{CM}+\mathrm{Cext}+\mathrm{Cpap}
$$

Em que: $C T=$ Custo total $\left(R \$ / m^{3} c c\right) ; C C=$ Custos de reconstrução $\left(R \$ / m^{3} c c\right) ; C M=$ Custos de manutenção $\left(R \$ / m^{3} c c\right) ; C e x t=$ Custos de extração $\left(R \$ / m^{3} c c\right) ; C$ pap $=$ Custos de perda de área produtiva $(R \$ /$ $\left.m^{3} c c\right)$.

A DOE, então, foi determinada como sendo o menor valor da curva de custo total resultante da relação desta com a densidade de estradas. Para o cálculo da DAE, considerou-se um intervalo 2\% acima do custo total mínimo, conforme Malinovski (2013). Por fim, foi determinado o espaçamento entre estradas (EE) para situação de DE, DOE e DAE, conforme a equação a seguir:

$$
\mathrm{EE}=\frac{10000}{\mathrm{DE} \text { ou DOE ou DAE }}
$$

Em que: $E E=$ Espaçamento entre estradas $(m) ; D E=$ Densidade atual de estradas $(\mathrm{m} / \mathrm{ha}) ; \mathrm{DOE}=$ Densidade ótima de estradas $(\mathrm{m} / \mathrm{ha}) ; \mathrm{DAE}=$ Densidade aceitável de estradas $(\mathrm{m} / \mathrm{ha})$.

Todas as informações e dados foram obtidos no período de janeiro a julho de 2016.

\section{RESULTADOS E DISCUSSÃO}

O custo total de reconstrução das estradas foi, em média, de $R$ \$32,48 por metro linear e $R$ \$ $32.480,00$ por quilômetro de estrada e, com operações de manutenção, em média, de $\mathrm{R} \$ 17$,56 por metro linear e R\$17.560,00 por quilômetro (Figura 1).
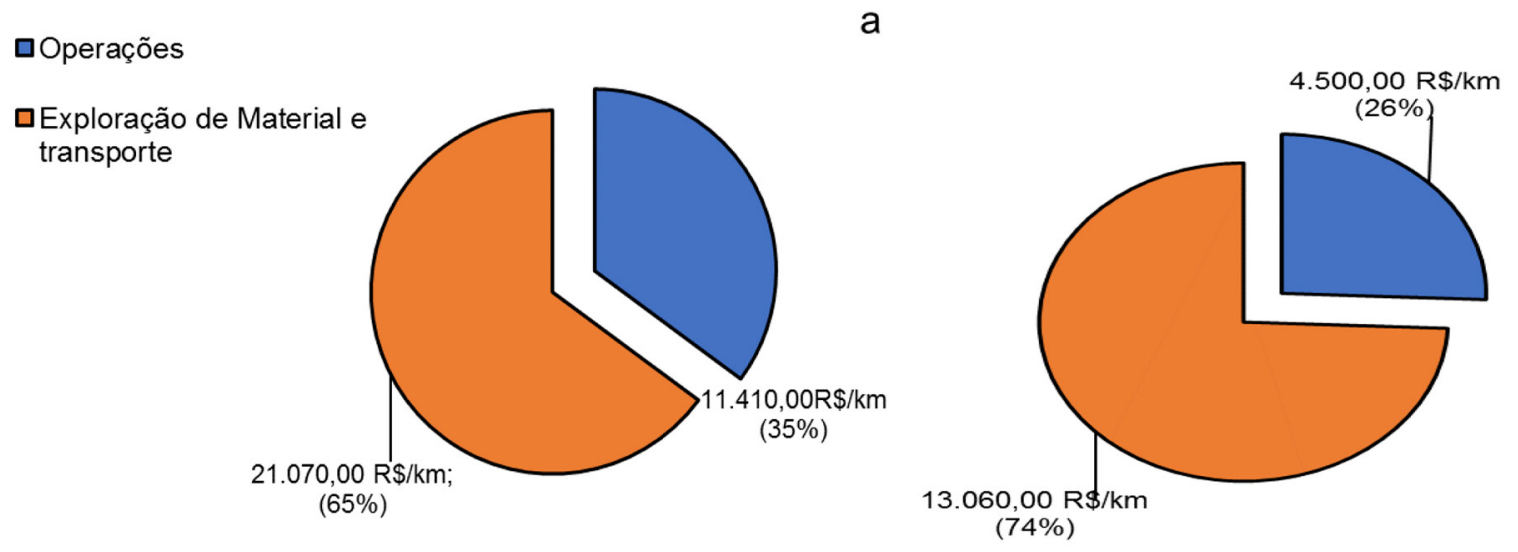

Figura 1. Custos em valores absolutos e em percentuais por atividade para as operações de reconstrução e manutenção de estradas.

Figure 1. Absolute and percentage costs per activity for the road reconstruction and maintenance operations.

Os custos relacionados às atividades de exploração e transporte de material rochoso corresponderam a maior porcentagem do custo total na operação de reconstrução, equivalendo a $65 \%$. De modo semelhante, verificou-se isso para as operações de manutenção, onde $74 \%$ do custo desta operação foram com atividades de exploração e transporte de material. A quantidade de material utilizada nesta área de corte foi de cerca de $1649 \mathrm{~m}^{3}$, sendo $47 \%$ em reconstrução e $53 \%$ em manutenção.

De modo geral o volume de material a ser utilizado em cada área depende de algumas variáveis, tais como: época do ano que será realizada a colheita, pois se realizada nas estações de inverno e primavera, em função da maior precipitação, necessitará de maior volume de material para dar condições ao transporte de madeira; tipo de material a ser utilizado, cada tipo de material tem uma capacidade de suporte diferente, sendo que o mais fraco necessita de maior espessura quando colocado na estrada, necessitando de maior volume do mesmo; volume de madeira a ser colhido, que dimensionará a quantidade de estradas que serão cascalhadas para fornecer acesso ao transporte de madeira; entre outros fatores.

Devido aos clientes transportarem as toras dentro das áreas da empresa, as estradas devem permitir o tráfego de veículos pesados e extrapesados mesmo em condições de declividade adversa e 
em dias de chuva, visto que a precipitação média anual da região é de $1800 \mathrm{~mm}$ (ALVAREZ et al., 2014) e o relevo é forte ondulado. Com isso, os custos de produção (Figura 2) são maiores ao encontrado nos trabalhos de Carmo et al. (2013), Souza (2011) e Zagonel et al. (2008).

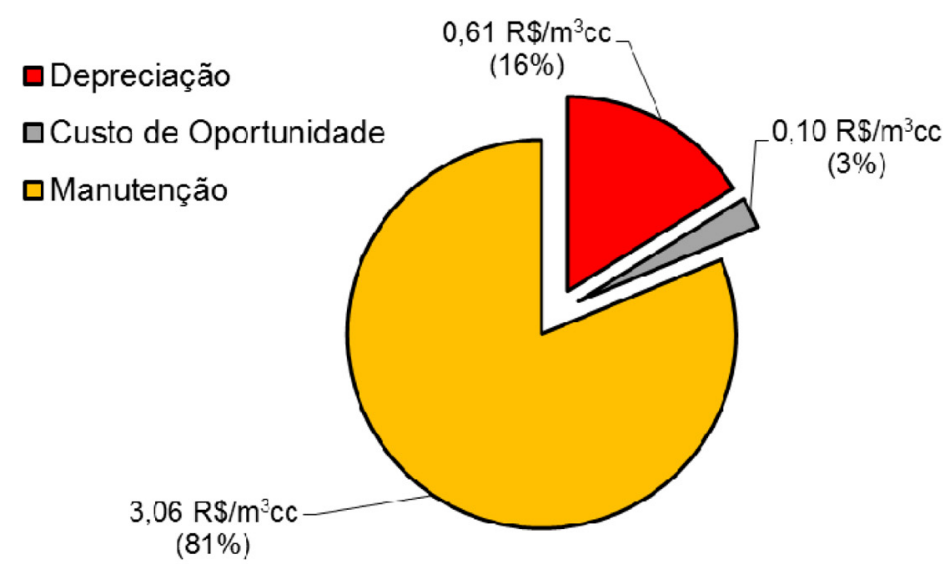

Figura 2. Custos de produção de estradas em valores absolutos e percentuais relacionados à depreciação, oportunidade do capital e de manutenção de estradas.

Figure 2. Road production costs in absolute and percentage values related to road depreciation, capital opportunity and maintenance.

Além disso, o alto valor de investimento em estradas na área de estudo foi devido às decisões estratégicas da empresa, pois o volume de madeira colhido em corte raso correspondia, em média, a $50 \%$ da receita mensal, assim, tal valor foi investido visando que o transporte ocorra em qualquer condição climática, uma vez que após a madeira ser colhida, a mesma deve ser transportada para sua utilização em até cinco dias, de modo a evitar o azulamento e consequente perda de qualidade da madeira, o que inviabilizaria a sua comercialização.

Em relação aos custos de perda de área produtiva considerando a situação atual da área, o custo era de $\mathrm{R} \$ 7,57 \mathrm{~m}^{3} / \mathrm{cc}$. Ao reduzir a área de estradas em função da DOE, o custo no sistema CTL passou a ser de $\mathrm{R} \$ 1,29 \mathrm{~m}^{3} / \mathrm{cc}$ e em sistema FT de $\mathrm{R} \$ 1,88 \mathrm{~m}^{3} / \mathrm{cc}$, correspondendo a um ganho de $2,38 \%$ e $2,16 \%$, respectivamente.

Quanto aos custos com extração da madeira, estes foram maiores no sistema de colheita CTL do que no sistema FT, independente da distância de extração (Figura 3).
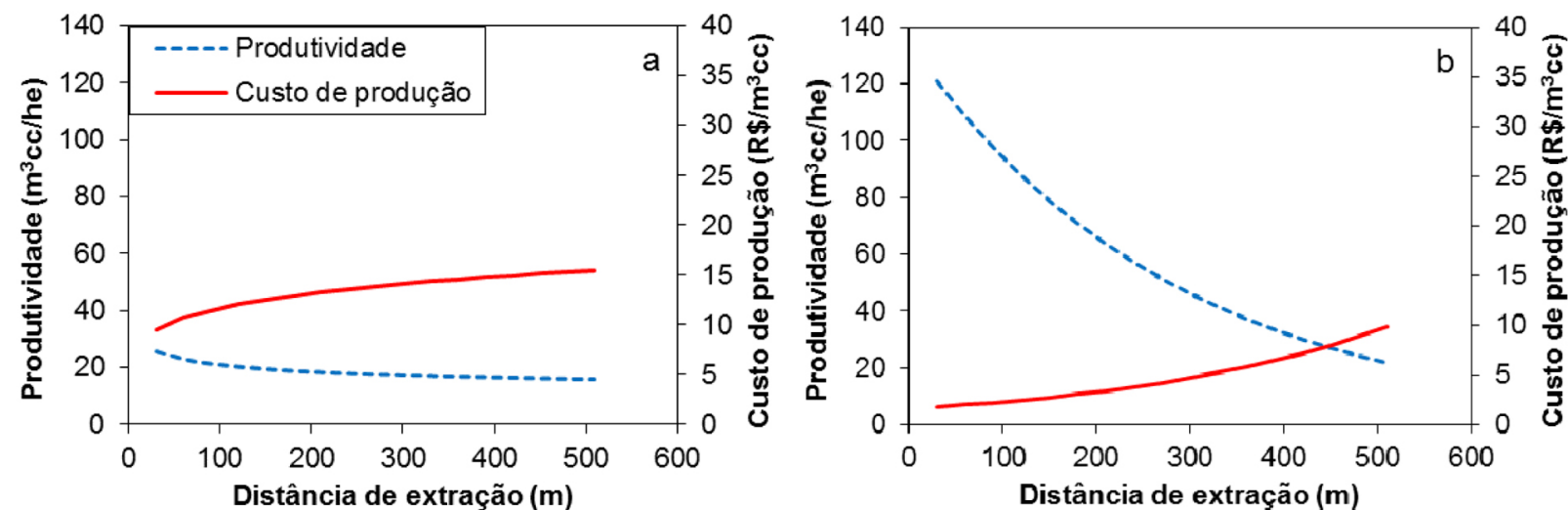

Figura 3. Relação entre produtividade e o custo de produção de operações de extração com a distância de extração para forwarder em sistema de colheita de toras curtas (a) e skidder em sistema de árvores inteiras (b) em corte raso de pinus.

Figure 3. Relationship between productivity and the production cost of extraction operations with the extraction distance for forwarder in cut-to-length harvesting system (a) and skidder in full tree harvesting system (b) in clear cut of pine.

Este resultado se deveu à menor produtividade do forwarder em relação ao skidder, pois, o primeiro equipamento possuía uma produtividade efetiva de $19,88 \mathrm{~m}^{3} \mathrm{cc} /$ he, já o segundo de $72,36 \mathrm{~m}^{3} \mathrm{cc} /$ he, refletindo em um maior custo de produção. 
Entretanto, é importante destacar que, com o aumento da distância de extração, a tendência de redução de produtividade foi maior para o skidder em relação ao forwarder. Essa diferença advém da forma em que cada máquina despende o seu tempo dentro do ciclo operacional, pois, em geral, o skidder consome maior parte do tempo do seu ciclo com atividades de deslocamento vazio e deslocamento carregado, conforme já encontrado nos trabalhos de Birro et al. (2002) e Seixas (2008). Enquanto que o forwarder consome maior tempo do ciclo com atividades de carregamento e descarregamento da madeira, concordando com o sugerido por Lopes et al. (2016), Seixas (2008) e Minette et al. (2004). Com isso, como se pode verificar, o aumento da distância de extração tendeu a aumentar o custo de produção de maneira mais expressiva para operação com skidder do que para a operação com forwarder, concordando com o sugerido por Seixas e Castro (2014).

No entanto, ainda vale salientar que existem alguns fatores que influenciam a operação de extração com o forwarder que não influenciam a operação com skidder e vice-versa. No caso do forwarder, fatores como o volume por tora, organização e número de sortimentos e quantidade de sortimentos baldeados simultaneamente por ciclo, interferem significativamente a produtividade operacional (MALINOVSKI et al., 2006; OLIVEIRA et al., 2009) diferentemente do skidder, que para o qual esses fatores não interferem sua produtividade operando em sistema de colheita de árvores inteiras.

Para o sistema CTL, a DOE foi de 15,63 m/ha, ou seja, um valor $83 \%$ menor que a DE avaliada na presente situação (Figura 4). Assim, com a utilização da mesma, pode haver uma redução de 77 $\mathrm{m} /$ ha de estradas nesta área, tendo disponível para plantio 132,76 ha e resultando numa economia de até $6,47 \mathrm{R} \$ / \mathrm{m}^{3} \mathrm{cc}$ do custo total (Tabela 2).

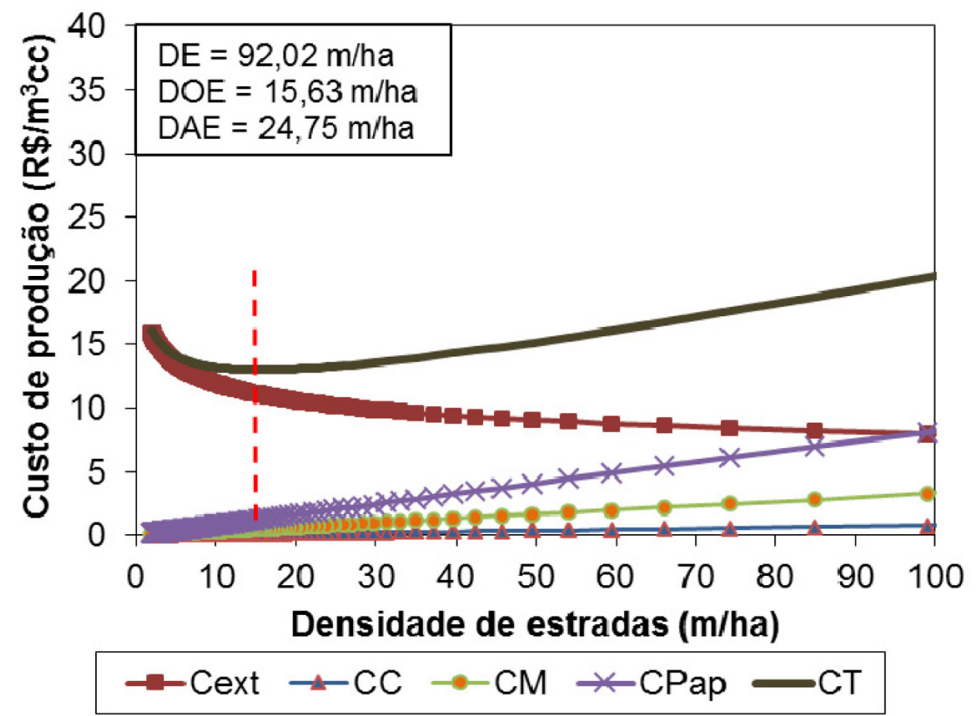

Figura 4. Relação entre a densidade de estradas e os custos de reconstrução (CC) e manutenção de estradas (CM), perda de área produtiva (Cpap), extração (Cext) e custo total (CT) para o corte raso em sistema de colheita de toras curtas. DE: densidade atual de estradas; DOE: densidade ótima de estradas; DAE: densidade aceitável de estradas.

Figure 4. Relationship between road density and costs of road reconstruction (CC) and maintenance (CM), productive area loss (Cpap), extraction (Cext) and total cost (CT) for the clearcutting in cut-to-length harvesting system. DE: current road density; DOE: optimal road density; DAE: acceptable road density.

A DOE para o sistema FT (Figura 5) foi de 22,85 m/ha, portanto, para se alcançar o ótimo em termos de malha viária, seria necessária uma redução de $75 \%$ na quantidade de estradas secundárias e terciárias na área, o que potencialmente acarretaria uma economia de $6,99 \mathrm{R} \$ / \mathrm{m}^{3} \mathrm{cc}$ de madeira produzida.

Comparando os dois sistemas de colheita avaliados, verificou-se que a quantidade ótima de estradas para operações de corte raso em sistema de árvores inteiras foi $46 \%$ superior em relação ao ótimo para o sistema de toras curtas. Isso se deveu às diferenças entre as operações de extração de cada sistema. Como já comentado, a distância de extração teve uma influência maior na produtividade e custos para operação de extração com skidder do que para a operação com forwarder, ocasionando na necessidade de maior quantidade de estradas para realização da extração no sistema FT. 


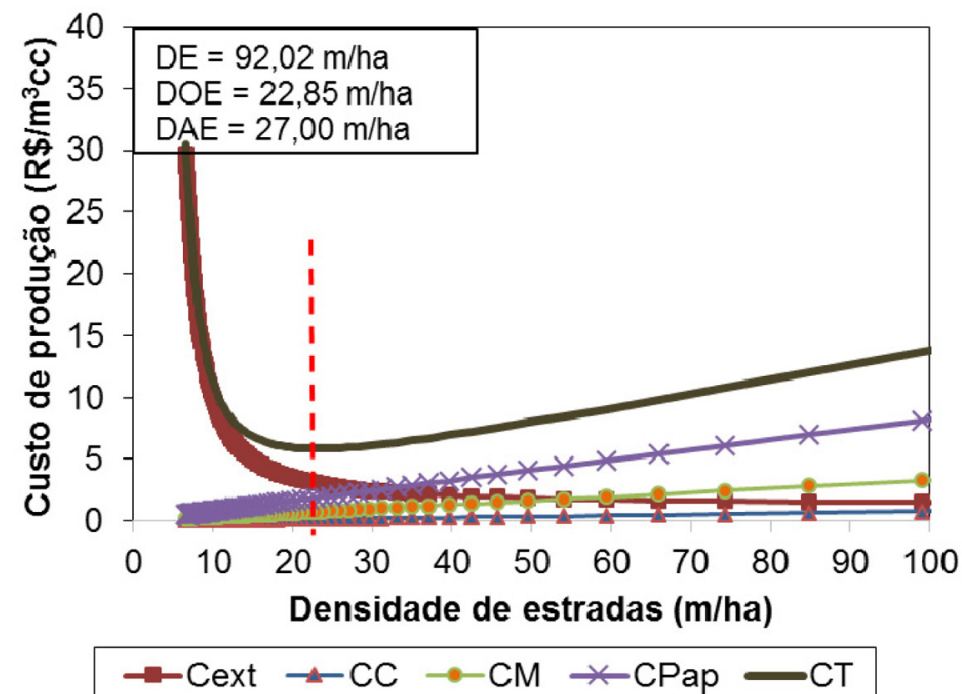

Figura 5. Relação entre a densidade de estradas e os custos de reconstrução (CC) e manutenção de estradas (CM), perda de área produtiva (Cpap), extração (Cext) e custo total (CT) para o corte raso em sistema de coIheita de árvores inteiras. DE: densidade atual de estradas; DOE: densidade ótima de estradas; DAE: densidade aceitável de estradas.

Figure 5. Relationship between road density and costs of road reconstruction (CC) and maintenance (CM), productive area loss (Cpap), extraction (Cext) and total cost (CT) for the clearcutting in full tree harvesting system. DE: current road density; DOE: optimal road density; DAE: acceptable road density.

Em relação à DAE, esta foi 9\% maior para o sistema FT do que para o sistema CTL, ou seja, uma diferença menor entre os sistemas do que a diferença de DOE. Em situações como a do presente estudo, onde já houveram investimentos e implantação da rede viária, além de restrições quanto às condições de relevo e onde os talhões apresentam geometria irregular e são descontinuados devido à áreas de preservação permanente e etc., a DAE pode ser utilizada como um parâmetro alternativo à $\mathrm{DOE}$, visto que, embora ambas sejam determinadas utilizando somente variáveis econômicas e não operacionais, muitas vezes, a distância média de extração e espaçamento entre estradas na DOE (Tabela 2) podem ser inviáveis operacionalmente.

Tabela 2. Custos médios de produção de reconstrução e manutenção de estradas, de extração e de perda de área produtiva para os valores atuais, ótimos e aceitáveis de densidade de estradas, de distância média de extração e de espaçamento entre estradas para o sistema de toras curtas e árvores inteiras.

Table 2. Mean production costs of road reconstruction and maintenance, extraction and productive area loss to the current, optimal and acceptable values of road density, mean extraction distance and road spacing for the cut-to-length and full tree systems.

\begin{tabular}{|c|c|c|c|c|c|c|c|c|c|}
\hline \multirow{3}{*}{ Situação } & \multirow{3}{*}{ Sistema } & \multicolumn{8}{|c|}{ Variáveis } \\
\hline & & CC & CM & Cext & Cpap & CT & DE & DME & EE \\
\hline & & & & $\mathrm{R} \$ / \mathrm{m}^{3} \mathrm{cc}$ & & & (m/ha) & (m) & (m) \\
\hline Atual & & 0,71 & 3,06 & 8,13 & 7,57 & 19,47 & 92,02 & 64,56 & 108,67 \\
\hline Ótima & CTL & 0,12 & 0,52 & 11,07 & 1,29 & 12,99 & 15,63 & 380,00 & 639,65 \\
\hline Aceitável & & 0,19 & 0,82 & 10,21 & 2,03 & 13,25 & 24,75 & 240,00 & 403,99 \\
\hline Atual & & 0,71 & 3,06 & 1,53 & 7,57 & 12,87 & 92,02 & 64,56 & 108,67 \\
\hline Ótima & FT & 0,18 & 0,76 & 3,07 & 1,88 & 5,88 & 22,85 & 260,00 & 437,65 \\
\hline Aceitável & & 0,21 & 0,90 & 2,67 & 2,22 & 6,00 & 27,00 & 220,00 & 370,32 \\
\hline
\end{tabular}

CTL: sistema de colheita de toras curtas; FT: sistema de colheita de árvores inteiras; CC: custo de reconstrução; CM: custo de manutenção; Cext: custo de extração; Cpap: custo de perda de área produtiva; CT: custo total; DE: densidade de estradas; DME: distância média de extração; EE: espaçamento entre estradas.

Por isso, o uso da DAE pode ser favorecido, uma vez que seu uso implicaria somente em um aumento de $2 \%$ do custo total. No presente caso, considerando a DAE, a vantagem que o sistema CTL traria em termos de redução de estradas e seus custos decorrentes em relação ao sistema FT, seria menor do que se utilizasse a DOE.

Na situação atual, em sistema CTL os custos com extração correspondem a $42 \%$ do custo total. Utilizando-se a DOE ou a DAE para este sistema, os custos com extração aumentarão chegando a representar 85 e 77\%, respectivamente, entretanto os custos com reconstrução e manutenção de 
estradas e, principalmente, com perda de área produtiva serão de aproximadamente quatro vezes menores. Assim, com o emprego da DOE será possível reduzir o custo total em até 33,26\%, já com o emprego da DAE essa redução pode ser de até 31,85\% (Figura 6).

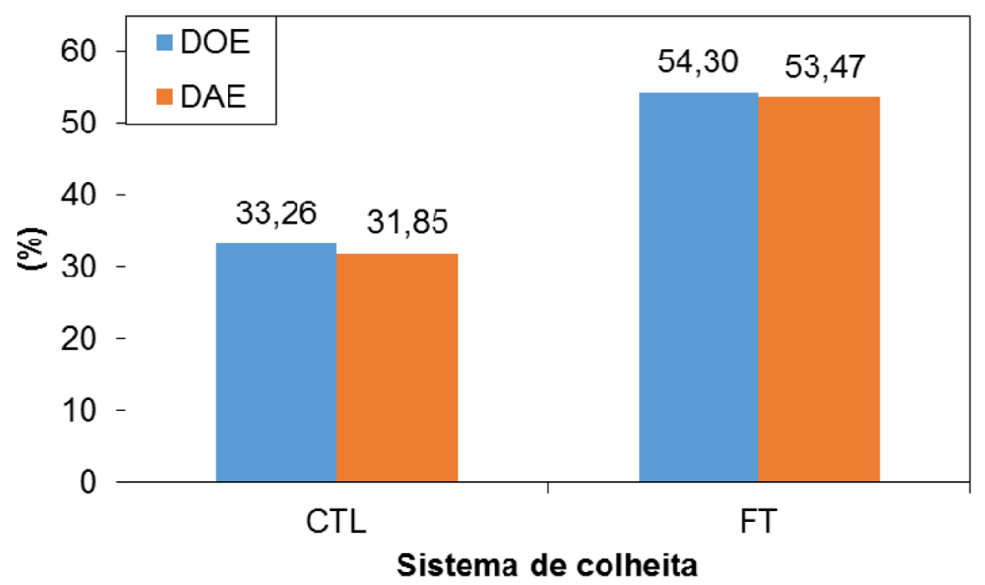

Figura 6. Percentagem de redução do custo total na densidade ótima (DOE) e aceitável (DAE) em comparação à densidade atual de estradas para o sistema de colheita de toras curtas (CTL) e árvores inteiras (FT).

Figure 6. Percentage reduction of the total cost in the optimal (DOE) and acceptable density (DAE) in comparison to the current of road density for the cut-to-length (CTL) and full tree (FT) harvesting systems.

Para o sistema FT, na DOE e na DAE os custos com extração poderão equivaler a 50\% do custo total. Enquanto os custos com estradas e perda de área produtiva também serão menores, em semelhança ao sistema CTL, porém, em menor magnitude (Tabela 1). Contudo, para este sistema caso se atinja a DOE ou DAE, a percentagem de redução do custo total será maior do que para o sistema CTL, podendo haver uma redução de 54,30 e 53,47\% do custo total na DOE e DAE, respectivamente (Figura 6).

Embora os custos de produção sejam menores para o sistema FT, ressalvas devem ser tomadas para o emprego desse sistema, pois, seu uso requer uma densidade maior de estradas em comparação com o CTL, como demonstrado pelos resultados. E com uma maior densidade de estradas, possivelmente os impactos ambientais e, consequentemente, os custos para mitigação destes impactos serão também maiores. Por isso, a escolha de um sistema de colheita deve ser pautada em sua viabilidade econômica e ambiental, além de suas características operacionais as quais devem atender aos critérios do manejo da floresta, visando a busca da sustentabilidade florestal.

Nesse sentido, a otimização da rede viária é uma alternativa estratégica importante para a redução nos custos de produção de matéria prima, investimentos em construção e manutenção de estradas e perda de áreas de efetivo plantio (ZAGONEL et al., 2008). Desta forma, observa-se a necessidade do replanejamento das estradas nessa área visando à otimização dos recursos e maximização das receitas, independentemente do sistema de colheita a ser empregado.

\section{CONCLUSÕES}

A densidade atual de estradas foi superior em relação às densidades ótima e aceitável para ambos os sistemas de colheita, demonstrando a necessidade do uso de critérios técnicos para planejamento da rede viária visando a redução de custos.

A densidade ótima e aceitável de estradas foi inferior no sistema de colheita de toras curtas em comparação ao sistema de colheita de árvores inteiras, sendo reflexo da diferença entre as operações de extração de madeira, pois no sistema de árvores inteiras, o skidder mostrou-se mais sensível ao aumento da distância de extração em relação ao forwarder no sistema de toras curtas.

Na densidade ótima ou aceitável de estradas, a redução de custos totais foi maior no sistema de colheita de árvores inteiras comparado ao sistema de toras curtas, em função da menor produtividade $\mathrm{e}$, consequentemente, maiores custos com operações de extração do sistema de toras curtas, embora os custos com reconstrução e manutenção e perda de área produtiva sejam menores para este sistema. 
Sampietro et al. - Densidade ótima e aceitável de estradas na

colheita de pinus no sistema de toras curtas e árvores inteiras

\section{AGRADECIMENTOS}

À empresa florestal pelo apoio na realização do trabalho e à FAPESC (Fundação de Amparo à Pesquisa e Inovação do Estado de Santa Catarina) pelo suporte financeiro ao Grupo de Pesquisa, Processo N. o 2017TR639"

\section{REFERÊNCIAS BIBLIOGRÁFICAS}

ALVAREZ, C. A.; STAPE, J. L.; SENTELHAS, P. C.; GONÇALVES, J. L.; SPAVOREK, G. Köppen's climate classification map for Brazil. Meteorologische Zeitschrift, Sttutgart, v. 22, n. 6, p. 711-728, 2014.

BIRRO, M. H. B; MACHADO, C. C.; SOUZA, A. P.; MINETTI, L. J. Avaliação técnica e econômica da extração de madeira de eucalipto com "track-skidder" em região montanhosa. Revista Árvore, Viçosa, v. 28, n. 2, p. 207-217, 2002.

CARMO, F. C. A.; FIEDLER, N. C.; LOPES, E. S.; PEREIRA, D. P.; MARIN, H. B.; SILVA, E. N. Análise da densidade ótima de estradas florestais em propriedades rurais. Cerne, Lavras, v. 19, n. 3, p. 451-459, 2013.

LOPES, E. S.; MACHADO, C. C.; SOUZA, A. P. Classificação e custos de estradas em florestas plantadas na região sudeste do Brasil. Revista Árvore, Viçosa, v. 26, n. 3, p. 329-338, 2002.

LOPES, E. S.; TONHATO, L.; RODRIGUES, C. K.; SERPE, E. L. Declividade do terreno e distância de extração na produtividade do forwarder com guincho de tração auxiliar. Nativa, v. 4, n. 6, p. 347-352, 2016.

MACHADO, C. C. Construção e conservação de estradas rurais e florestais. Viçosa: UFV, 2013. 441 p.

MALINOVSKI, R. A. Densidades aceitáveis de estradas. In: ENCONTRO BRASILEIRO DE INFRAESTRUTURA FLORESTAL, 1., 2013, Curitiba. Anais... Curitiba: EBIF, 2013.

MALINOVSKI, R. A.; MALINOVSKI, R. A.; MALINOVSKI, J. R. YAMAJI, F. M. Análise das variáveis de influência na produtividade das máquinas de colheita de madeira em função das características físicas do terreno, do povoamento e do planejamento operacional florestal. Floresta, Curitiba, v. 36, n. 2, p. 169-182, 2006.

MINETTE, L.; MOREIRA, F. M.; SOUZA, A. P.; MACHADO, C. C.; SILVA, K. R. Análise técnica e econômica do forwarder em três subsistemas de colheita de florestas de eucalipto. Revista Árvore, Viçosa, v. 28, n. 1, p. 91-97, 2004.

OLIVEIRA, D.; LOPES, E. S.; FIEDLER, N. C. Avaliação técnica e econômica do Forwarder na extração de toras de pinus. Scientia Forestalis, Piracicaba, v. 37, n. 84, p. 525-533, 2009.

RYAN, T.; PHILLIPS, H.; RAMSAY, J.; DEMPSEY, J. Forest Road Manual: Guidelines for the design, construction and management of forest roads. Dublin: COFORD, 2004. 170 p.

SEIXAS, F. Extração florestal. In: MACHADO, C. C. Colheita florestal. 2. ed. Viçosa: UFV, 2008. p. 97-145.

SEIXAS, F.; CASTRO, G. P. Extração. In: MACHADO, C. C. Colheita florestal. 3. ed. Viçosa: UFV, 2014. p. 106-177.

SOUZA, C. M. G. Densidade ótima de estradas para povoamentos de teca (Tectona grandis L.f.) no estado de Mato Grosso. 2011. 55 p. Dissertação (Mestrado em Ciências Florestais e Ambientais) - Universidade Federal de Mato Grosso, Cuiabá, 2011.

USDA - UNITED STATES DEPARTMENT OF AGRICULTURE. Commodity Costs and Returns Measurement Methods. Washington: USDA, 2001. 566 p.

ZAGONEL, R.; CORRÊA, C. M. C.; MALINOVSKI, J. R. Densidade ótima de estradas de uso florestal em áreas de relevo plano em povoamentos de Pinus taeda no planalto catarinense. Scientia Forestalis, Piracicaba, v. 36, n. 77, p. 33-41, 2008.

Recebido em 06/02/2017

Aceito em 31/10/2017 